\title{
MULTIVARIABLE NONLINEAR MODEL REFERENCE CONTROL OF CEMENT MILLS
}

\author{
Mehmet Önder Efe ${ }^{1}$ and Okyay Kaynak ${ }^{2}$ \\ ${ }^{1}$ Carnegie Mellon University, Electrical and Computer Engineering Department \\ Pittsburgh, PA 15213-3890, U.S.A., efemond@andrew.cmu.edu \\ ${ }^{2}$ Bogazici University, Electrical and Electronic Engineering Department \\ Bebek 80815, Istanbul, Turkey, kaynak@boun.edu.tr
}

\begin{abstract}
This paper presents a method for model reference control of a cement-milling circuit that has been studied previously. The approach presented is based on the experimentally justified model developed by Breugesen (1996), and derives the form of control vector with the goal of driving the behavior of the system to that of a desired model. The derivation is based on the Lyapunov theory, and the results observed confirm the tracking claims of the paper. Copyright $\odot 2002$ IFAC
\end{abstract}

Keywords: Nonlinear Control, Model Reference Control, Industrial Control, Lyapunov Stability

\section{INTRODUCTION}

Control of industrial processes has been the focus a number of research studies. The techniques studied in the area of nonlinear control have successfully been applied and some of which have been exemplified in Breusegen et al (1996); Magni et al (1999), Grognard et al (2001), Dagci et al (2001), Clarke (1988) and Thomas (1991). Particularly the model used in this paper has constituted a prime example due to the strong interdependencies between the variables involved. The model has three state variables and two control inputs, despite its representational simplicity, the dynamics is quite complex and a good control performance can only be achieved if a suitable coordination between the control inputs can be established.

The first results on this system have been presented in Breusegen (1996), which is based on on-site experimentation of the system, and which constitute a basis for the dynamic model presented in Magni et al (1999). It has been shown in Breusegen et al (1996) that linear quadratic control scheme based on the minimization of several system specific performance criteria could lead to an admissible results. In Magni et al (1999), the multivariable predictive control of the system is studied. The problems associated with the plugging phenomenon and a robust control scheme is studied by Grognard et al (2001), one prime conclusion reported in which is the necessity to include mill load in the state feedback information. Dagci et al (2001) have used the same model to test the control performance of a sliding mode based design approach. It has been observed that an acceptable response could be obtained using the setpoint values given in Magni et al (1999).

Although the model studied in this paper constitutes a good example to test the performance of novel control schemes, in the literature, various other studies focusing on cement mills have appeared. Clarke (1988) discusses the predictive control technique for industrial processes including a cement mill and adopts a receding horizon approach. Since the process performance depends heavily on the physical properties of the mill drives, in Thomas (1991) these issues are addressed.

This paper is organized as follows: The second section describes briefly the dynamics of the cement milling circuit. The adopted reference model is introduced in the third section and the synthesis of the control signals is presented in the fourth section. In the fifth section, we formulate the error dynamics 
for all three states when the observations are noisy. The sixth section is devoted to the justification of the proposed scheme and the concluding remarks are given at the end of the paper.

\section{DYNAMICS OF THE CEMENT-MILLING CIRCUIT}

The dynamic model of the system is described by three coupled and nonlinear differential equations as given in (1)-(3). Briefly, $z$ is the mill load, $y_{f}$ is the product flow rate and $y_{r}$ is the tailings flow rate. These three variables are the states of the system. On the other hand, $\varphi$ is the output flow rate of the mill and denotes the relative hardness of the material inside the mill with respect to the nominal one, which is unity. The system has two control inputs, denoted by $u$, feed flow rate and, $v$, classifier speed.

$$
\begin{aligned}
& \dot{z}=-\varphi(z, d)+u+y_{r} \\
& T_{f} \dot{y}_{f}=-y_{f}+(1-\alpha(z, v, d)) \varphi(z, d) \\
& T_{r} \dot{y}_{r}=-y_{r}+\alpha(z, v, d) \varphi(z, d)
\end{aligned}
$$

where the functions $\alpha(z, v, d)=\frac{\varphi(z, d)^{m} v^{n}}{K_{\alpha}+\varphi(z, d)^{m} v^{n}}$, and $\varphi(z, d)=\max \left(0,\left(-d K_{\varphi 1} z^{2}+K_{\varphi 2} z\right)\right) . K_{\alpha}=570^{m} 170^{n}(570 / 450-$ 1). $(\text { Tons } / \mathrm{h})^{\mathrm{m}} \mathrm{r} / \mathrm{min}^{\mathrm{n}}, m=0.8, n=4, K_{\varphi 1}=0.1116$ (Tons $* \mathrm{~h})^{-1}, K_{\varphi 2}=16.50 \mathrm{~h}^{-1}, T_{f}=0.3 \mathrm{~h}, T_{r}=0.01 \mathrm{~h}$ and $d=$ 1. A schematic representation of the process is depicted in Fig. 1, and a detailed description of the system dynamics and results regarding the experimental verification can be found in Breusegen et al (1996) and Magni et al (1999).

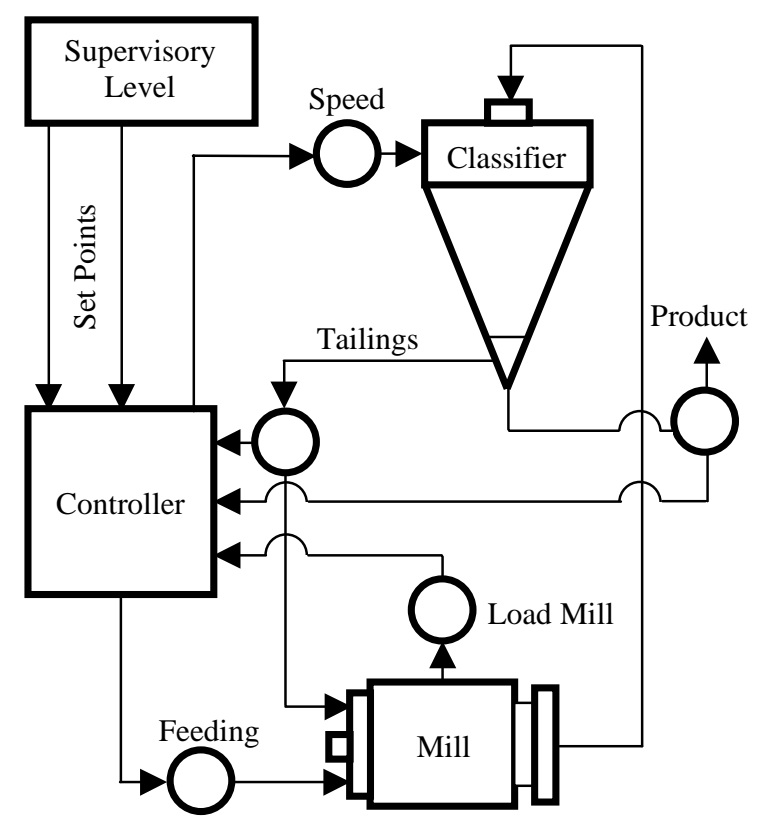

Fig. 1. Schematic Diagram of the Cement Milling Circuit

The control problem is to enforce the system states by appropriately altering the two control inputs. However, it can easily be shown that the designer can choose two of the three state variables independently as the behavior of the third state variable will be determined upon the selection of other two. It is emphasized in Breusegen et al (1996) that the choice of $y_{f}$ and $y_{r}$ may lead to unachievable values for $\varphi$ and it is suggested that keeping $y_{f}$ and $z$ under control would be a suitable approach. In this paper, we adopt the same reasoning and proceed parallel to this idea.

\section{REFERENCE MODEL}

In choosing the reference dynamics for $y_{f}$ and $z$, we consider the following design requirements.

- Reference model for each state, whose variables are represented with a subscript $m$, must be stable and must follow the command signal.

- The speed of response imposed by the reference system must not be higher than that of the actual system, i.e., the time constants must be compatible.

$\dot{z}_{m}=-z_{m}+r$

$T_{f} \dot{y}_{m f}=-y_{m f}+f$

where $r$ and $f$ are the command signals for $z_{m}$ and $y_{m f}$.

\section{SYNTHESIS OF THE CONTROL SIGNAL}

Assume the third state variable $\left(y_{m r}\right)$ of the model has the following dynamics, with $Q$ being a real valued smooth function of the state variables, command signals and system variables.

$T_{r} \dot{y}_{m r}=Q$

Consider the following Lyapunov function candidate,

$V=\frac{1}{2} e_{z}^{2}+\frac{T_{f}}{2} e_{y f}^{2}+\frac{T_{r}}{2} e_{y r}^{2}$

where

$e_{z}=z-z_{m}$

$e_{y f}=y_{f}-y_{m f}$

$e_{y r}=y_{r}-y_{m r}$

Evaluating the time derivative of the Lyapunov function in (7) yields the following.

$\dot{V}=e_{z}\left(\dot{z}-\dot{z}_{m}\right)+T_{f} e_{y f}\left(\dot{y}_{f}-\dot{y}_{m f}\right)+T_{r} e_{y r}\left(\dot{y}_{r}-\dot{y}_{m r}\right)$

Inserting (1)-(3) and (4)-(6) into (11) gives the following quantity.

$$
\begin{aligned}
\dot{V}= & e_{z}\left(-\varphi+u+y_{r}+z_{m}-r\right)+ \\
& e_{y f}\left(-y_{f}+(1-\alpha) \varphi+y_{m f}-f\right)+ \\
& e_{y r}\left(-y_{r}+\alpha \varphi-Q\right)
\end{aligned}
$$


One suitable selection for $u$ and $v$ inputs can be solved by imposing the following:

$$
\begin{aligned}
& -\varphi+u+y_{r}+z_{m}-r=-e_{z} \\
& -y_{f}+(1-\alpha) \varphi+y_{m f}-f=-e_{y f}
\end{aligned}
$$

The two equalities in (13) lead to the following time derivative for the Lyapunov function, furthermore, the control inputs can now be formulated as given in (15) and (16).

$$
\begin{aligned}
& \dot{V}=-e_{z}^{2}-e_{y f}^{2}+e_{y r}\left(-y_{r}+\alpha \varphi-Q\right) \\
& u=\varphi-y_{r}-z+r \\
& v=K_{\alpha}^{1 / n}\left(\frac{\varphi}{f}-1\right)^{1 / n} \varphi^{-m / n}
\end{aligned}
$$

Once the explicit forms of the control signals $u$ and $v$ are obtained, we need to find out the third component of the reference dynamics, which is imposed by the selected control law. It can mathematically be proved that the formulated control laws in (15) and (16) lead to the truth of following equalities:

$$
\alpha=1-\frac{f}{\varphi}
$$

Or equivalently,

$$
\alpha \varphi=\varphi-f
$$

Requiring $-y_{r}+\alpha \varphi-Q=-e_{y r}+\varphi-\varphi_{m}$ and using (18) lead to $Q=-y_{m r}+\varphi_{m}-f$. More explicitly, the dynamics of the $y_{r}$ state is driven towards the $y_{m r}$ dynamics of the mode given by

$$
T_{r} \dot{y}_{m r}=-y_{m r}+\varphi_{m}\left(z_{m}, d\right)-f
$$

However, now we can rewrite (14) as follows

$$
\dot{V}=-e_{z}^{2}-e_{y f}^{2}-e_{y r}^{2}+T_{r} e_{y r}\left(\varphi-\varphi_{m}\right)
$$

Assuming the hardness parameter $d$ is available for model update, the selection of $u$ given in (15) forces $z \rightarrow z_{m}$, hence $\varphi \rightarrow \varphi_{m}$. Furthermore, with the selected control inputs, the states $y_{f}$ and $z$ are decoupled and are enforced to follow the corresponding model variables. The result in (20) has therefore the following meaning: During initial transient period there "may" be an increase in tailings flow rate, however, this regime will not last long due to the derived model and the constructed control strategy.

\section{ANALYSIS WITH NOISY OBSERVATIONS}

In this section, we analyze the consequences of the noisy observations. For this purpose, assume that the states of the system are corrupted by a noise sequence additively, and denote the noisy sequences with $\zeta_{z}, \zeta_{y f}$ and $\zeta_{y r}$. The control signals of (15) and (16) will have the following forms:

$$
\begin{aligned}
& u=\varphi\left(z+\zeta_{z}, d\right)-\left(y_{r}+\xi_{y r}\right)-\left(z+\zeta_{z}\right)+r \\
& v=K_{\alpha}^{1 / n}\left(\frac{\varphi\left(z+\zeta_{z}\right)}{f}-1\right)^{1 / n} \varphi\left(z+\zeta_{z}\right)^{-m / n}
\end{aligned}
$$

Application of the above controls to the system of (1)-(3), and denoting $\varphi_{\zeta}=\varphi\left(z+\zeta_{z}, d\right)$, the model following errors of (8)-(10) are obtained as follows:

$$
\begin{aligned}
& \dot{e}_{z}=-e_{z}+\varepsilon_{1} \\
& T_{f} \dot{e}_{y f}=-e_{y f}+\varepsilon_{2} \\
& T_{r} \dot{e}_{y r}=-e_{y r}+\varepsilon_{3}
\end{aligned}
$$

where

$$
\begin{aligned}
& \varepsilon_{1}=-\left(\zeta_{y r}+\zeta_{z}\right)+\left(\varphi_{\zeta}-\varphi\right) \\
& \varepsilon_{2}=\frac{\varphi}{1+\left(\frac{\varphi}{\varphi_{\zeta}}\right)^{m}\left(\frac{\varphi_{\zeta}}{f}-1\right)}-f
\end{aligned}
$$

$$
\varepsilon_{3}=\frac{\varphi\left(\frac{\varphi}{\varphi_{\zeta}}\right)^{m}\left(\frac{\varphi_{\zeta}}{f}-1\right)}{1+\left(\frac{\varphi}{\varphi_{\zeta}}\right)^{m}\left(\frac{\varphi_{\zeta}}{f}-1\right)}-\varphi_{m}+f
$$

Clearly, if $\left\|\zeta_{z} \quad \zeta_{y f} \quad \zeta_{y r}\right\| \rightarrow 0$, then $\varphi_{\zeta} \rightarrow \varphi$. The implications of this are as follows $\varepsilon_{1} \rightarrow 0, \varepsilon_{2} \rightarrow 0$ and $\varepsilon_{3} \rightarrow \varphi-\varphi_{m}$. For the term in (28), as we have already observed in (20), the model following error in the tailings flow rate $\left(e_{y r}\right)$ converges to zero as $z \rightarrow z_{m}$. In the next section, we present the simulation studies.

\section{JUSTIFICATION OF THE PROPOSED LAW - SIMULATIONS}

During the simulations, the plant is kept under an ordinary feedback loop, and the control signals are generated using the noisy observations of the system response. The disturbance corrupting the $z$ state of the system has variance equal to 1.8278 and that corrupting the state $y_{r}$ has variance equal to 1.7062 . The maximum amplitude of the noise sequences is equal to 5 and both sequences have zero means and are Gaussian distributed. Clearly, the given disturbance scenario stipulate that the state variables required to construct the control signals are severely corrupted. We set the simulation stepsize to $0.005 \mathrm{~h}$ and simulated the control system for 90 hours.

Initially $y_{f}(0)=216, y_{r}(0)=0$ and $z(0)=99$, on the other hand, the reference model states have initially been 
set to $y_{m f}(0)=168, y_{m r}(0)=20$ and $z_{m}(0)=77$. In the upper subplot of Fig. 2, the command signal $(r)$, the response of the reference model $\left(z_{m}\right)$ and the response of the system are illustrated together. Approximately after 5 hours, the model output and the plant output become almost indistinguishable. In order to clarify the tracking claim of the paper, the lower subplot depicts the model following error in state $z$.

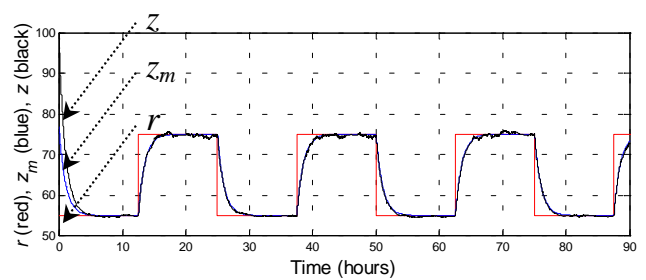

Model Following Error for $Z$

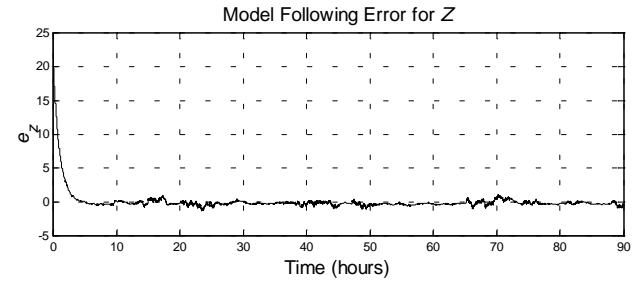

Fig. 2. The command signal ( $r$, the response of the reference model and the observed mill load behavior.

Similarly for the product flow rate $\left(y_{f}\right)$, in the top row of Fig. 3, the command signal ( $f$, which is independent of $r$ ) and the response of the reference model $\left(y_{m f}\right)$ and the response of the system are illustrated. The model following error is shown in the bottom row of the figure. It is apparent that the error quickly converges to zero and remains in the close neighborhood of zero.
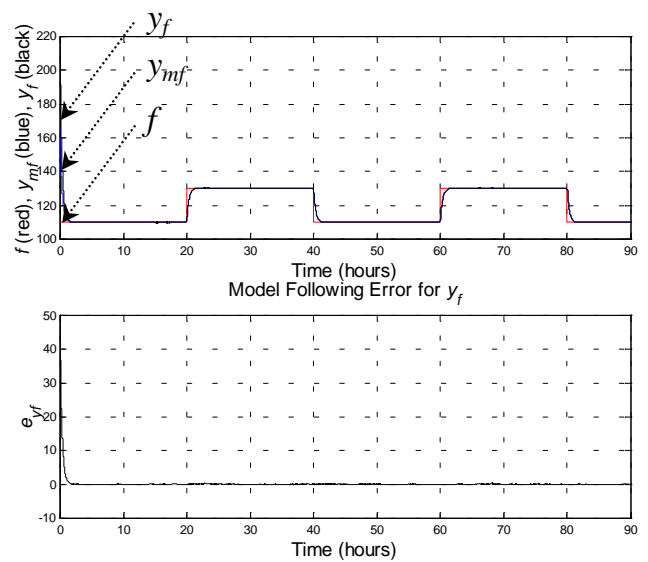

Fig. 3. The command signal $(f)$, the response of the reference model and the observed product flow rate.

The results regarding the third state $\left(y_{r}\right)$ are visualized in Fig. 4. The bottom suplot of the figure demonstrates that the model following error comes close to zero after a fast transient effective during the initial phase.

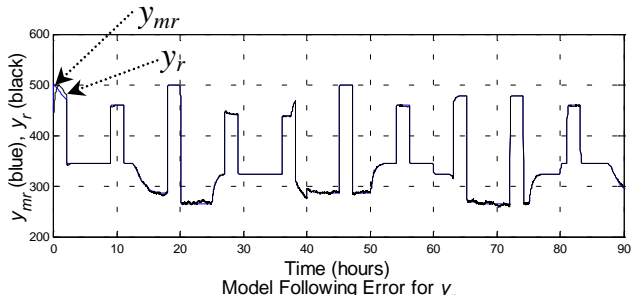

Model Following Error for $y$

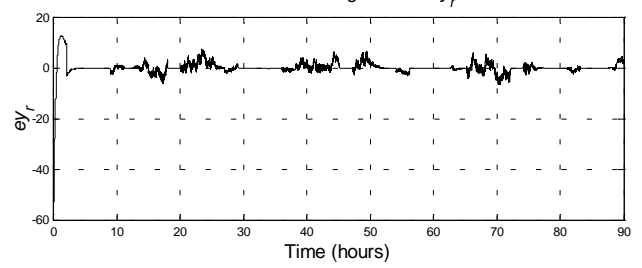

Fig. 4. The response of the reference model $\left(y_{m r}\right)$ and that of the milling circuit.

The applied control signals are depicted in Fig. 5, which suggests that the scheme is reasonably safe in terms of the control signal magnitudes. Although there seems to be high frequency components in the ontrol signal, the reader must notice that the horizontal axis is in hours, and the state variables are corrupted. Therefore, observing such a behavior in the shown scale is parallel to what is imposed by the operating conditions.
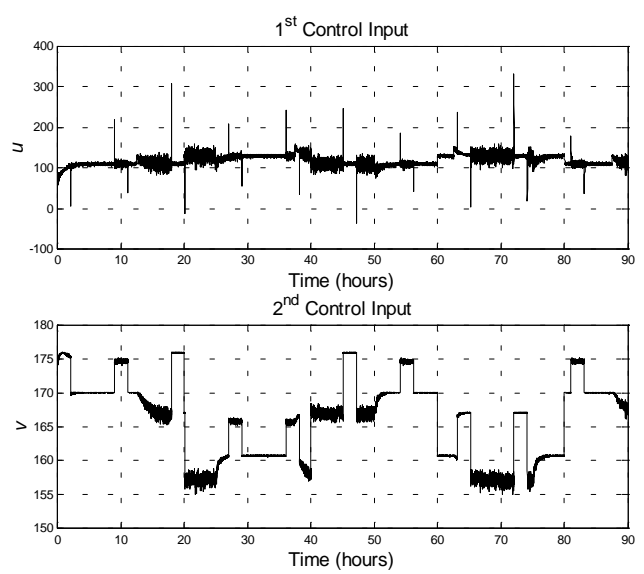

Fig. 5. Applied control signals, $u$ at the upper subplot and $v$ at the lower subplot.

Another important result that should be emphasized is observed in the behavior of the Lyapunov function of (7). In Fig. 6, three subplots are given. The top one and the middle one depict the behavior of the Lyapunov function for the entire course of the simulation except the top one is in linear and the middle one in logarithmic time axis. Apparently, the results confirm the negative definiteness of the time derivative of the selected Lyapunov function is observed as a result of the adopted control strategy. The lowest subplot of Fig. 6 illustrates the behavior of $V$ after 2.8 hours. The instantaneous spikes are due to the corrupted state information used by the controller. When the noise magnitudes, which were equal to five and the maximal magnitude of the 
spikes seen in this subplot are compared, it can directly be inferred that the control system is capable of handling the disturbances.
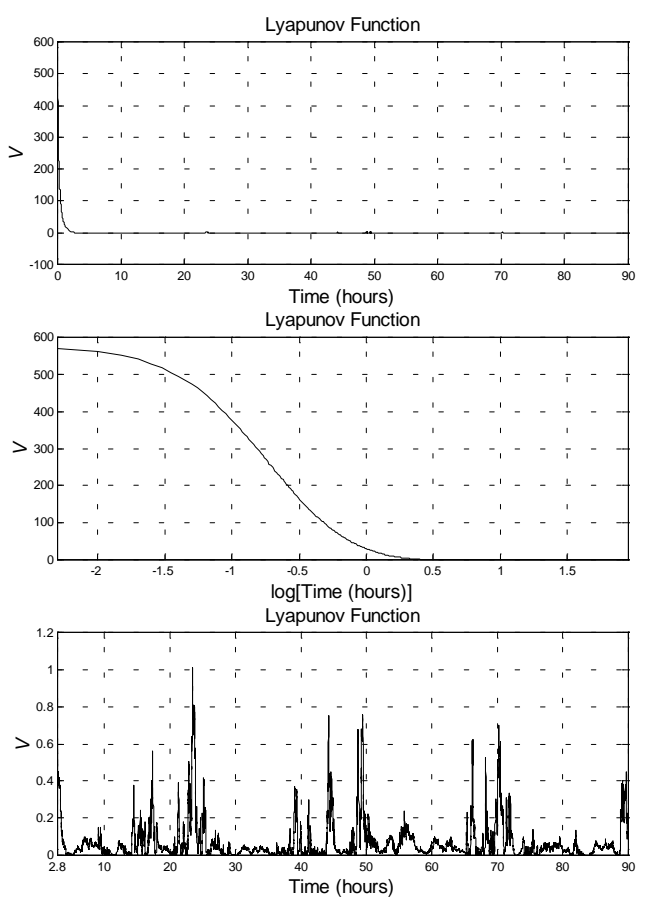

Fig. 6. The behavior of Lyapunov function in (7) with linear time axis (upper subplot), with logarithmic time axis (middle subplot) and with linear time axis but after 2.8 hours.

Another important reason that make the problem challenging is the abrupt variations in the material hardness parameter $(d$, whose behavior is illustrated in Fig. 7. The hardness of the material inside the mill has a time-varying behavior as shown in Fig. 7. As studied in Magni et al (1999), we change the value of $\mathrm{d}$ from unity to 1.34 , but we have adopted this change periodic. Referring to the discussion in section 3, we have claimed that the constructed control signals would enforce $z \rightarrow z_{m}$ and hence $\varphi(z, d) \rightarrow \varphi_{m}\left(z_{m}, d\right)$. In Fig. 8, we illustrate the behavior of $\varphi_{m} / \varphi$, which confirms the above quoted convergence statement. Apparently the tendency of this quantity is to lie around unity.

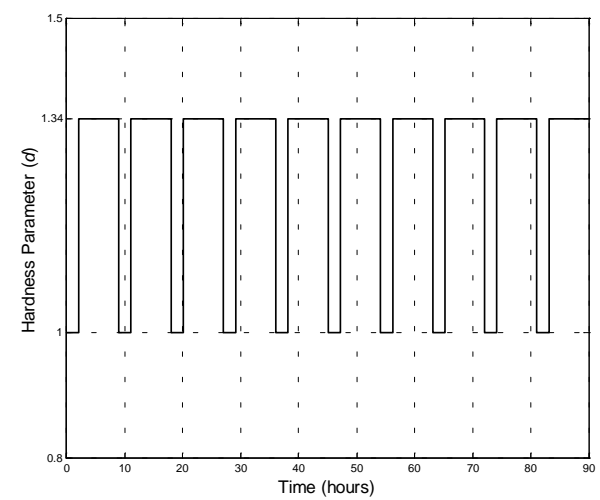

Fig. 7. The change in the hardness of the material in the mill $(d)$.

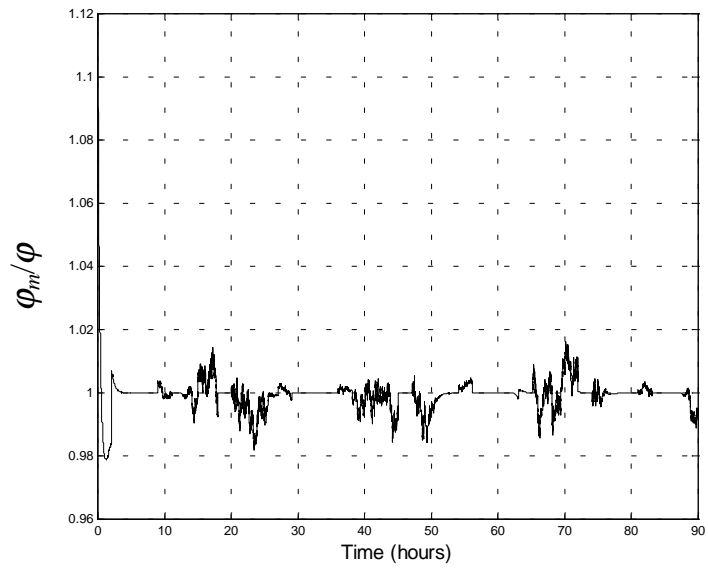

Fig. 8. Behavior of the quantity $\varphi_{m} / \varphi$, which is maintained around unity.

Lastly in Fig. 9, we focus on the imposed and observed behaviors of the mill load $\left(z_{m}\right.$ and $\left.z\right)$ in the three dimensional space spanned by time, $\varphi$ (or $\varphi_{m}$ ) and mill load. This figure clarifies that the change in the hardness parameter enforces a motion on the surface described by the new value of the parameter $d$, i.e. $\varphi\left(z, d_{\text {new }}\right)$ surface. When the response of the reference model and that of the milling circuit are drawn together, it becomes more apparent that the trajectory imposed on this space is followed by the cement mill system. The dark black trajectory in this figure depicts the behavior of $z_{m}$ and the gray colored trajectory is for $z$.

The two trajectories are almost indistinguishable, and this observation suggests that the formulated control signals drive the behavior of the mill load to what is prescribed by the reference model.

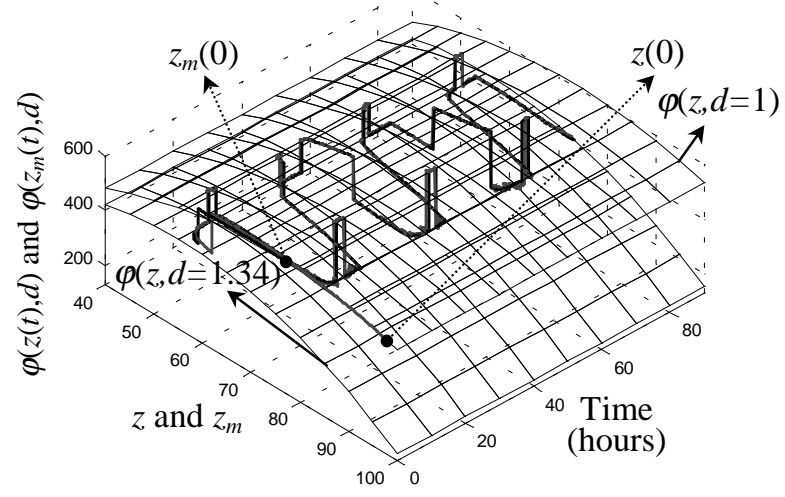

Fig. 9. The behavior of the mill load $\left(z\right.$ and $\left.z_{m}\right)$ in three dimensional space with two surfaces described by $\varphi(z(t) \in[40,100], d=1) \quad$ and $\varphi(z(t) \in[40,100], d=1.34)$

\section{CONCLUSIONS}

A nonlinear control strategy for a cement milling process is studied. The constructed forms of the control signals have resulted in good performance in 
terms of the model following capability, disturbance rejection and robustness.

Considerable amount of observation noise, large initial errors and response with time varying system parameters are studied and it has been observed that the suggested strategy results in good performance in terms of all these control specific metrics.

On the other hand, one limitation of what is developed is the fact that it necessitates the dynamic model of the plant under control. Secondly, the model has to be updated as the hardness parameter (d) changes. Our future work aims to reformulate the control signals such that the analytical details can intelligently be constructed and the value of the parameter $d$ can be estimated from the state evolution of the plant.

\section{REFERENCES}

Breusegen, V.V., L. Chen, G. Bastin, V. Wertz, V. Werbrouck and C. de Pierpont (1996). An industrial application of multivariable linear quadratic control to a cement mill circuit, IEEE Transactions on Industrial Applications, v.32, pp.670-677.

Clarke, D.W. (1988). Application of generalized predictive control to industrial processes, IEEE Control Systems Magazine, v.8, no.2, pp.49-55.

Dagci, O.H., M.O. Efe, and O. Kaynak (2001). A nonlinear learning control approach for a cement milling process, to appear in Proc. 2001 IEEE Conf. on Control Applications (CCA) and 2001 IEEE Int. Symposium on Intelligent Control (ISIC), September 5 - 7, Mexico City, Mexico, 2001.

Grognard, F., F. Jadot, L. Magni, R. Sepulchre, and V. Wertz (2001). Robust stabilization of a nonlinear cement mill model, IEEE Transactions on Automatic Control, v.46, no.4, pp.618-623.

Magni, L., G. Bastin and V. Wertz (1999). Multivariable nonlinear predictive control of cement mills, IEEE Transactions on Control Systems Technology, v.7, no.5, pp.502-508.

Thomas, P.F. (1991). Development of mill drives for the cement industry, Cement Industry Technical Conference, XXXIII, pp.101-117. 